$63^{\text {ème }}$ Congrès de la SFCO, 02015 (2015)

DOI:10.1051/sfco/20156302015

(C) Owned by the authors, published by EDP Sciences, 2015

\title{
COMMUNICATION
}

\section{Carcinome pulmonaire neuroendocrine à grandes cellules métastatique révélé par une hyperplasie gingivale}

\author{
Souron $\mathrm{B}^{* * * *}$, Schultz $\mathrm{P}^{* * * * * *}$, Weingertner $\mathrm{N}^{* * * * * * * *}$, Chenard $\mathrm{M}-\mathrm{P} * * * * * * * *$, \\ Goichot $\mathrm{B}^{* * * * * * * * *}$, Missler $\mathrm{J} * * * * * * *$, Kurtz J-E**,********, Féki A $\mathrm{A}^{* * * * *}$, Bornert \\ $\mathrm{F}^{*}, * * * *$ \\ * Unité de Chirurgie buccale, CHU de Strasbourg \\ ** Faculté de Médecine, Université de Strasbourg \\ *** Service d'ORL, CHU de Strasbourg \\ **** Faculté de Chirurgie Dentaire, Université de Strasbourg \\ ***** Service d'Anatomopathologie, CHU de Strasbourg \\ ****** Service de Médecine Interne, CHU de Strasbourg \\ ******* Service de Pneumologie, hôpital de Sarrebourg \\ ******** Service d'Oncologie, CHU de Strasbourg
}

Introduction. Les tumeurs neuroendocrines sont des tumeurs rares qui dérivent du système neuroendocrine diffus. Elles peuvent donc apparaître dans tous les organes. La classification 2004 de l'OMS qui concerne les tumeurs neuroendocrines pulmonaires, les répartit en 4 groupes : carcinoïde typique, carcinoïde atypique, carcinome neuroendocrine à petites cellules et carcinome neuroendocrine à grandes cellules.

Présentation du cas. Nous rapportons le cas d'un patient de 70 ans adressé par son dentiste pour une lésion gingivale hyperplasique autour de la dent 27. Il présentait comme antécédent un adénocarcinome pulmonaire avec métastase pleurale, diagnostiqué 18 mois auparavant et traité par chimiothérapie. La biopsie de la lésion buccale a conduit au diagnostic de carcinome neuroendocrine à grandes cellules. L'analyse immunohistochimique révélait une positivité pour CD56, synaptophysine, chromogranine et CK7, et une négativité pour TTF-1 et CK20. Le cone-beam ne montrait pas d'extension osseuse au maxillaire. Pour identifier le site primitif, un TEP-TDM au 18-FDG a été réalisé : il retrouvait une hyperfixation au niveau pulmonaire, pleural gauche, ganglionnaire médiastinal, cervical et rétro-péritonéal, buccal, hépatique, surrénalien gauche et osseux. Le diagnostic d'adénocarcinome pulmonaire connu avait été posé sur l'analyse cytologique du liquide pleural. La bronchoscopie et les études immunohistochimiques n'ont pas été contributives. Nous avons conclu à une métastase buccale d'un carcinome pulmonaire neuroendocrine à grandes cellules.

Discussion. Le diagnostic de carcinome neuroendocrine à grandes cellules repose sur des critères morphologiques et immunohistochimiques. Les tumeurs neuroendocrines à grandes cellules de la cavité orale, qu'elles soient primitives ou secondaires, sont extrêmement rares. L'analyse histologique ne permet pas de déterminer si une lésion est primitive ou secondaire. C'est pourquoi des études immunohistochimiques ont été développées avec l'exploration de nouveaux outils d'orientation diagnostique comme TTF-1 ou l'association CK7/CK20. Dans notre cas, la lésion intrabuccale est apparue dix-huit mois après la découverte de l'adénocarcinome pulmonaire. La littérature rapporte que les carcinomes pulmonaires neuroendocrines à grandes cellules opérés avaient été initialement mal diagnostiqués à la biopsie initiale, étiquetés le plus souvent adénocarcinomes. Par ailleurs, il existe des

This is an Open Access article distributed under the terms of the Creative Commons Attribution License 4.0, which permits unrestricted use, distribution, and reproduction in any medium, provided the original work is properly cited. 
carcinomes neuroendocrines à grandes cellules composites, avec un contingent neuroendocrine et un contingent non neuroendocrine. L'adénocarcinome est le contingent non neuroendocrine le plus fréquent. Pour aller plus loin, il semblerait que le phénotype neuroendocrine puisse apparaître secondairement au cours de l'évolution d'adénocarcinomes au stade métastatique. Il n'y a pas de consensus de traitement pour les carcinomes pulmonaires neuroendocrines à grandes cellules. La chimiothérapie administrée dans le traitement des carcinomes pulmonaires à petites cellules ne donne pas de résultat probant pour ce type de cancer à un stade avancé. Des thérapies moléculaires ciblées sont en cours d'évaluation.

Conclusion. A notre connaissance, il s'agit du premier cas de métastase buccale d'un carcinome neuroendocrine à grandes cellules décrit dans la littérature anglaise. Les mécanismes biologiques et physiopathologiques nécessitent une meilleure compréhension pour aboutir à un traitement qui puisse améliorer le pronostic.

Nom et adresse du conférencier

Bornert FABIEN

Département de chirurgie buccale, Hôpitaux Universitaires de Strasbourg

1 place de l'hôpital

Pôle de Médecine et de Chirurgie bucco-dentaire

CHRU Strasbourg

67000 Strasbourg (France)

fabien.bornert@chru-strasbourg.fr 